PRAXIS

educativa

Universidad Nacional de La Pampa

Facultad de Ciencias Humanas

Instituto de Ciencias de la Educación

para la investigación interdisciplinaria

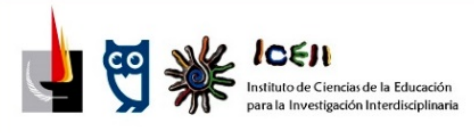

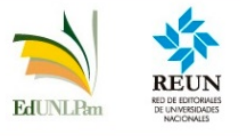

ISSN 2313-934X

SANTA ROSA, LA PAMPA, ARGENTINA

Correo electrónico: iceii@humanas.unlpam.edu.ar

Disponible en https://cerac.unlpam.edu.ar/index.php/praxis

La Ley Yolanda, un avance hacia la alfabetización ambiental y la educación en desarrollo sostenible en Argentina. Artículo de Alan Vogelfanger y María Virginia Mas. Praxis educativa, Vol. 25, No 3 septiembre - diciembre 2021. E - ISSN 2313-934X. pp. 1-16. https://dx.doi.org/10.19137/praxiseducativa-2021-250316

Esta obra se publica baja Licencia Creative Commons 4.0 Internacional

CC BY- NC-SA Atribución, No Comercial, Compartir igual

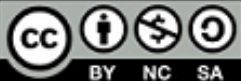

\title{
La Ley Yolanda, un avance hacia la alfabetización ambiental y la educación en desarrollo sostenible en Argentina
}

The Yolanda Law, a step forward for the education for sustainable development in Argentina

Lei Yolanda, um avanço na alfabetização ambiental e na educação para o desenvolvimento sustentável na Argentina

\section{Alan Vogelfanger}

Facultad de Filosofía y Letras, Facultad de Derecho. Universidad de Buenos Aires

Argentina

avogelfanger@gmail.com

ORCID: 0000-0002-8339-4767

\section{María Virginia Mas}

Facultad de Bioquímica y Ciencias Biológicas, Universidad Nacional del Litoral

Argentina

virmas12@gmail.com

ORCID: 0000-0002-8780-8728

Recibido: 2021- 03-16 | Revisado: 2021-05-03 | Aceptado: 2021-05- 03 


\title{
Resumen
}

El Congreso de la Nación argentino promulgó, en diciembre de 2020, la Ley Yolanda, que impone capacitaciones obligatorias en materia de ambiente, con perspectiva de desarrollo sostenible y con énfasis en cambio climático, para las personas que trabajan en la función pública. El propósito de este artículo es aprovechar la adopción de esta norma, que establece que todos los empleados y todas las empleadas del Estado deberán tener una formación ambiental, para recordar los orígenes y debates actuales que giran en torno a este tema, remarcar su vínculo con la educación en derechos humanos y señalar las obligaciones gubernamentales de garantizar que toda la población adquiera estos aprendizajes. Asimismo, se destacarán los principales puntos de la ley y de los debates en las Cámaras de Senadores y Diputados. Se concluirá que se trata de un paso positivo para lograr la alfabetización ambiental, aunque aún quede mucho camino por recorrer.

Palabras clave: alfabetización ambiental; educación ambiental; educación en desarrollo sostenible; educación en derechos humanos; cambio climático.

\begin{abstract}
In December 2020, the Argentinean Congress enacted Law No 27.592, also known as "Yolanda Law". This norm imposes a mandatory training on environment, sustainable development and climate change for every public servant. The purpose of this article is to take this new law as a trigger to discuss the origins and current debates on environmental education, to define its relationship with human rights education, and to clarify the State obligation to guarantee that every person acquires this kind of education. Furthermore, the article will explain the main aspects of the Yolanda Law and it will highlight the most relevant speeches of the debates in both of the Chambers of the Congress. It will conclude by stating that the norm is a step forward towards achieving an environmental education for all, though we still have a long way to go.
\end{abstract}

Keywords: environmental literacy; environmental education; education for sustainable development; human rights education; climate change.

\section{Resumo}

O Congresso argentino promulgou, em dezembro de 2020, a Lei Yolanda, que impõe a formação obrigatória em questões ambientais, com uma perspectiva de desenvolvimento sustentável e com ênfase nas mudanças climáticas, para pessoas que exerçam funções públicas. O objetivo deste artigo é aproveitar a adoção dessa norma, que estabelece que todos os funcionários do Estado devem ter uma formação ambiental, para relembrar as origens e os debates atuais em torno ao tema, destacar sua vinculação com a educação em direitos humanos e indicar as obrigações estatais para garantir que toda a população adquira tal aprendizagem. Da mesma forma, se destacarão os principais pontos da lei e dos debates no Senado e na Câmara dos Deputados. Conclui-se que este é um passo positivo para a alfabetização ambiental, embora ainda haja um longo caminho a percorrer.

Palavras-chave: alfabetização ambiental; educação ambiental; educação para o desenvolvimento sustentável; educação para os direitos humanos; mudança climática. 


\section{Introducción}

En los últimos tiempos, el ambiente, concebido como todos los seres vivos que habitan en él (incluidos los seres humanos) y las relaciones que se producen entre ellos, está sufriendo numerosas contrariedades: cambio climático, pérdida de biodiversidad, sobreexplotación de recursos, contaminación y enfermedades. Estos problemas pueden afectar la vida de las personas y poner en riesgo la efectivización de sus derechos humanos y muestran la urgente necesidad de trabajar en la construcción de una sociedad más justa, equitativa y sustentable para todos los seres que habitan el planeta hoy y los que lo harán en el futuro. Por eso, esta crisis requiere de apremiantes reformas políticas, económicas, vitales y educativas (Morin, 2011).

En este sentido, es preciso que la población sea capaz de reconocer las problemáticas ambientales, tomar decisiones basadas en información confiable y participar activamente en la solución de las mismas, es decir, que cuente con una correcta alfabetización ambiental. Entonces, la educación es una de las vías necesarias para iniciar la metamorfosis hacia este tipo de sociedades que se pretende alcanzar (Morin, 2011). Al mismo tiempo, es preciso el establecimiento de políticas públicas congruentes con ello.

En relación con esto, la sanción de la Ley Yolanda en Argentina (Ley № 27.592), en diciembre de 2020, confirma la decisión de avanzar en este camino, ya que impone una capacitación obligatoria sobre ambiente y desarrollo sostenible para todas las personas que trabajan en la función pública. ¿Cómo se ha llegado a este punto? ¿Cuál ha sido el desarrollo de la educación en materia ambiental y en derechos humanos en nuestro país? ¿Qué propone la Ley Yolanda? ¿Cuáles fueron los debates en las Cámaras de Senadores y Diputados que lograron que fuera promulgada? Este artículo pretende hacer una revisión de estos interrogantes y de la historia de la Educación Ambiental para analizar las políticas públicas que la República Argentina ha elaborado al respecto a lo largo del tiempo hasta llegar a la mencionada ley. Asimismo, se hará una breve referencia a la Ley de Educación Ambiental Integral, sancionada por el Congreso, al momento de finalizarse este artículo.

\section{Educación ambiental y Alfabetización Ambiental}

La educación ambiental (EA) puede ser entendida como una compleja y esencial dimensión de la educación global (Sauvé, 2010). Es una educación para toda la vida, que puede desarrollarse tanto en el ámbito formal como en el no formal, que considera al "ambiente en su totalidad (natural, y el humano, ecológico, político, económico, social, legal, cultural y estético), que afectará a todas las áreas del conocimiento (transversalidad)" (González Gaudiano, 2012, p. 17). Su objetivo es la construcción de una ciudadanía responsable, desde una mirada esperanzadora y no catastrófica, capaz de tomar decisiones fundamentadas en información confiable y participar en la solución de las problemáticas para asegurar la calidad ambiental (Mas y Vignolo, 2017).

Por otro lado, la alfabetización ambiental es un primer contacto para formar sociedades solidarias, responsables, críticas y activas. Se entiende a la alfabetización como posibilidad de 
aprender a pensar, de liberar la conciencia, y apunta a formar una ciudadanía capaz de participar en el ejercicio político frente a las soluciones de las problemáticas (Vilches y Gil Pérez, 2012; Telias et al., 2014). Dicho concepto fue usado por primera vez por Roth (1992), quien menciona que abarca cuatro componentes: conocimientos, afectos (actitudes y valores), habilidades y comportamientos ambientales.

Una persona alfabetizada ambientalmente reconoce los problemas, se preocupa por ellos, entiende la información de los expertos, la evalúa, decide consciente y éticamente y actúa en consecuencia (Pesis, 2015). Estos valores y actitudes deben fortalecerse durante toda la vida. Por ello, es necesario el sostenimiento de la EA como proceso global, profundo y amplio en todos los ámbitos.

Para lograr la alfabetización ambiental de la población, la escuela es imprescindible. Aunque no es el único eslabón. También se necesita de la familia, los medios de comunicación, las políticas públicas. Las prácticas educativas que buscan la alfabetización ambiental de la ciudadanía requieren del tratamiento de problemáticas ambientales cotidianas, locales y situadas que posibiliten a los educandos tomar decisiones sobre asuntos que afectan a su vida diaria. Además, no deben abordarse de manera fragmentada, necesitan obligadamente del entrecruzamiento de saberes, de la interdisciplinariedad. Precisan de la formación en saberes y competencias éticas, críticas y políticas (Sauvé, 2013).

\section{Su nexo necesario con la educación en derechos humanos}

La temática ambiental y la de los derechos humanos son claramente complementarias. De hecho, su interacción se hace cada vez más explícita en los foros internacionales. La iniciativa mundial más actual y de mayor envergadura, que probablemente mejor refleja la relación entre estos conceptos, es la Agenda 2030. Este programa, adoptado en 2015 desde la ONU, incluye 17 Objetivos de Desarrollo Sostenible (ODS) que abarcan numerosas metas y una guía común para todos los países. Los propósitos generales incluyen erradicar la pobreza, proteger el planeta y asegurar la prosperidad para todas las personas. Específicamente, la meta 4.7, que se enmarca en el objetivo específico sobre Educación de Calidad, sostiene que los Estados deben:

Asegurar que todos los alumnos adquieran los conocimientos teóricos y prácticos necesarios para promover el desarrollo sostenible, entre otras cosas mediante la educación para el desarrollo sostenible y los estilos de vida sostenibles, los derechos humanos, la igualdad de género, la promoción de una cultura de paz y no violencia, la ciudadanía mundial y la valoración de la diversidad cultural y la contribución de la cultura al desarrollo sostenible. (Asamblea General de las Naciones Unidas, 2015, p. 20)

Además, tribunales internacionales, como la Corte Interamericana de Derechos Humanos, han sostenido recientemente que "existe un amplio reconocimiento sobre la relación interdependiente entre la protección al medio ambiente, el desarrollo sostenible y los derechos humanos" (Corte IDH, 2017, párr. 52; Corte IDH, 2020, párr. 85). La Comisión Interamericana de Derechos Humanos (2019), por ejemplo, también sostuvo específicamente que "la relación estrecha 
entre los derechos humanos, el desarrollo sostenible y el medio ambiente abarca innumerables facetas y alcances" (CIDH, 2019, p. 35). Es decir, en la actualidad, no es posible imaginar la protección de los derechos humanos sin incluir el ambiente; y viceversa, la protección del ambiente debe beneficiarse del enfoque de derechos y del derecho internacional de los derechos humanos.

En este sentido, el derecho a un medio ambiente sano es un derecho humano por sí mismo, autónomo, pero, además, se trata prácticamente de un requisito para la satisfacción de los demás derechos. Es decir, "todos los derechos humanos dependen en última instancia de una biosfera saludable" (Relator Especial sobre los derechos humanos y el medio ambiente, 2020, p. 2) porque la degradación ambiental puede impedir que se den las condiciones que son el sustento para la existencia misma (Carmona Lara, 2010). En particular, la crisis ambiental puede afectar derechos humanos como el derecho a la vida, a la vivienda, al agua, a la alimentación, a la integridad personal, a la salud, a la propiedad, a la libertad de expresión y al acceso a la información, entre otros. A su vez, los efectos y las consecuencias de la crisis ambiental repercuten desproporcionadamente en grupos como niños, niñas y adolescentes, mujeres, personas que viven en situación de extrema pobreza, personas con discapacidad y pueblos indígenas, quienes requieren una protección adicional por conformar grupos en situación de vulnerabilidad. En síntesis, la prosperidad y la mejora de las vidas en nuestro planeta requieren de un trabajo en conjunto que involucre a ambas aristas: la ambiental y la de derechos humanos.

Precisamente, el marco de la educación es un espacio donde ambas iniciativas deben existir, complementarse y potenciarse. Si bien las iniciativas sobre educación en derechos humanos existen desde mucho antes, fue en 2011 que la Asamblea General de las Naciones Unidas adoptó una de las definiciones más aceptadas en la actualidad (Tibbitts, 2017). En su Declaración de las Naciones Unidas sobre educación y formación en materia de derechos humanos (2011), indicó que:

1. La educación y la formación en materia de derechos humanos están integradas por el conjunto de actividades educativas y de formación, información, sensibilización y aprendizaje que tienen por objeto promover el respeto universal y efectivo de todos los derechos humanos y las libertades fundamentales, contribuyendo así, entre otras cosas, a la prevención de los abusos y violaciones de los derechos humanos al proporcionar a las personas conocimientos, capacidades y comprensión y desarrollar sus actitudes y comportamientos para que puedan contribuir a la creación y promoción de una cultura universal de derechos humanos.

2. La educación y la formación en materia de derechos humanos engloban: a) La educación sobre los derechos humanos, que incluye facilitar el conocimiento y la comprensión de las normas y principios de derechos humanos, los valores que los sostienen y los mecanismos que los protegen; b) La educación por medio de los derechos humanos, que incluye aprender y enseñar respetando los derechos de los educadores y los educandos; c) La educación para los derechos humanos, que incluye facultar a las personas para que disfruten de sus derechos y los ejerzan, y respeten y defiendan los de los demás. 
De esta definición, se desprende el objeto de promover el respeto de los derechos humanos, prevenir abusos y vulneraciones, e impulsar una cultura de derechos humanos. Asimismo, se menciona el concepto de educación sobre derechos (aspecto temático, de contenido), por medio de o a través de los derechos humanos (enseñar con el ejemplo, coherencia), y para los derechos humanos (propósito transformador, de incidencia, influencia). Estos objetivos son compartidos por la educación ambiental, cuando se habla de fomentar la construcción de una ciudadanía responsable y estimular una mirada esperanzadora. La educación en derechos humanos igualmente debe ser una educación para toda la vida, que se desarrolle de forma transversal e interdisciplinaria en todos los niveles del ámbito formal, pero también en los espacios de educación no formal o informal, y debe enseñarse considerando las problemáticas cotidianas, locales y situadas (Pinto, 2010; IIDH, 2013; Ronconi, 2017; Medici, 2018).

En otras palabras, tanto la alfabetización ambiental como la educación en derechos humanos buscan estimular el pensamiento propio y liberar la conciencia; formar sociedades solidarias, responsables, críticas y activas; e impulsar y modificar ciertas prácticas cotidianas para elevar la calidad de vida de las personas (Fisas, 1998; Magendzo, 2001; Pérez Aguirre, 2007). A su vez, ambas iniciativas encuentran claras influencias y antecedentes en las enseñanzas de Paulo Freire. No se debe perder oportunidad de recordar, entonces, la estrecha interrelación entre estas propuestas y destacar las posibles sinergias que pueden conseguir si se trabajan a la par.

\section{Breve recorrido sobre la educación ambiental}

La educación ambiental (EA) ha atravesado, a lo largo de la historia, un extenso proceso de constitución. Si bien los debates ambientales surgen en los años sesenta con las denuncias de algunas Organizaciones No Gubernamentales y el libro publicado por Raquel Carson en 1962, Primavera Silenciosa, recién se enfatiza la necesidad del desarrollo de la EA desde la Primera Conferencia de las Naciones Unidas sobre el Medio Humano, Primera Cumbre de la Tierra, celebrada en 1972 en Estocolmo (Sauvé, 2003).

En 1975, se formuló el Programa Internacional de Educación Ambiental bajo la conducción de la Organización de las Naciones Unidas para la Educación, la Ciencia y la Cultura (UNESCO) y el Programa de las Naciones Unidas para el Medio Ambiente (PNUMA). También, en ese año, se organizó en Belgrado, Yugoslavia, el Seminario Internacional sobre Educación Ambiental y se establecieron sus metas y objetivos en la Carta de Belgrado. Este documento establece las pautas principales para la EA, como que debe considerar al ambiente en todos sus aspectos, que debe ser un proceso continuo, permanente, adoptar un método interdisciplinario y de participación activa (García y Priotto, 2009). Luego, en 1977, se realizó la Primera Conferencia Intergubernamental de EA en Tbilisi (Georgia), donde la EA quedó reducida a una herramienta de superación de problemas ambientales, que prepara a los alumnos para resolverlos, pero sin tratar sus causas de fondo.

Posteriormente, UNESCO y PNUMA convocaron, en 1987, en Moscú, al Congreso Mundial sobre Educación y Formación relativos al Medio Ambiente, con el objeto de hacer un balance del desarrollo de la EA y aprobar una estrategia internacional para los años noventa, declarando así la 
década 1990-2000 como el "Decenio Mundial de la Educación Ambiental". En 1987, también se publicó el Informe Bruntland, donde se instaló el término sostenibilidad, aludiendo a la preservación de los recursos de la naturaleza para las generaciones futuras. Desde entonces y a la fecha, se han realizado incontables congresos, simposios y conferencias, talleres de trabajo y mesas de gestión.

En 1992, se desarrolló la Segunda Cumbre de la Tierra, Conferencia Mundial sobre Ambiente y Desarrollo (CNUMAD), en Río de Janeiro (ECO 92 o Río 92). Sus estrategias se documentaron en la Agenda 21. A partir de esta Cumbre, aumentaron las leyes generales marco de ambiente promoviendo la implementación de la EA y se propuso un enfoque más global basado en la EA para el Desarrollo sostenible. De hecho, uno de los momentos claves de este desplazamiento conceptual de educación ambiental a educación en desarrollo sostenible es la Conferencia Internacional sobre Medio Ambiente y Sociedad: Educación y Sensibilización para la Sostenibilidad, celebrada en Thessaloniki (Salónica o Tesalónica) en 1997.

En 2002, por su parte, se celebró, en Sudáfrica, Johannesburgo, la Tercera Cumbre de la Tierra (conocida como "Río+10") y, en ese mismo año, desde Naciones Unidas y principalmente UNESCO, se adoptó el "Decenio de la Educación para el Desarrollo Sostenible", entre 2005 y 2014. En 2012, se efectuó la Conferencia de desarrollo sostenible de Naciones Unidas conocida como Río+20, en Río de Janeiro, Brasil. En ella, se trabajó sobre cómo construir una economía ecológica para lograr el desarrollo sostenible y sobre cómo mejorar la coordinación internacional para alcanzarlo (Báez Tobar, 2015). Como resultado de esta última reunión, se redactó el Acuerdo de Escazú que es un tratado internacional denominado Acuerdo Regional sobre el Acceso a la Información, la Participación Pública y el Acceso a la Justicia en Asuntos Ambientales en América Latina y el Caribe. El mismo fue adoptado en Escazú (Costa Rica) el 4 de marzo de 2018 y de él participaron 24 países. Desde entonces, comenzó el proceso de ratificación de los países suscriptores. Al haber alcanzado los requisitos necesarios (11 Estados parte, entre ellos, Argentina), el acuerdo entró en vigor el 22 de abril de 2021.

El Acuerdo de Escazú es el primer acuerdo regional ambiental de América Latina y el Caribe que establece garantías sobre el acceso a la información, la participación pública y el acceso a la justicia en asuntos ambientales. Dentro de sus objetivos, se encuentran: garantizar la implementación plena y efectiva de los derechos, sobre todo de los grupos vulnerables, luchar contra la desigualdad y la discriminación, "así como la creación y el fortalecimiento de las capacidades y la cooperación, contribuyendo a la protección del derecho de cada persona, de las generaciones presentes y futuras, a vivir en un medio ambiente sano y al desarrollo sostenible" (CEPAL, 2018, p. 14). Más aún, el Artículo 12 del Acuerdo señala, en particular, que los gobiernos deberán cooperar para adoptar medidas que mejoren la educación, la formación y la sensibilización del público sobre el cambio climático. La Ley Yolanda, de alguna manera, viene a cumplir o implementar parte de esta obligación, y lo mismo se puede decir sobre la Ley de Educación Ambiental Integral. 


\section{Estado de la cuestión sobre la educación ambiental en Argentina}

Particularmente, en Argentina, en 1973, se creó la Secretaría de Estado de Recursos Naturales y Ambiente Humano. Luego, no hubo avances en la temática debido a la dictadura militar que duró desde 1976 hasta 1983. Recién en 1990 surgió el Consejo Federal de Medio Ambiente (COFEMA) como espacio de encuentro federal para abordar los problemas y las soluciones del ambiente de la República Argentina, por iniciativa de las provincias que veían la necesidad de instalar el tema entre las políticas públicas nacionales, dada la urgencia. Los objetivos del COFEMA son formular políticas ambientales, coordinar estrategias y programas de gestión, exigir estudios de impacto ambiental, propiciar programas y acciones de educación ambiental, formar un banco de datos y proyectos ambientales y gestionar el financiamiento de los mismos. El COFEMA se propone facilitar una dinámica en red entre las provincias para la toma de decisión consensuada. Sin embargo, según Nogeira (2016), el funcionamiento de dicho Consejo solo ha consistido en algunas reuniones de discusión y pocas acciones concretas. Al mismo tiempo, también es cierto que el COFEMA, por ejemplo, impulsó fuertemente la Ley Yolanda, apoyó y generó campañas como "La escuela se planta ante el cambio climático" por la que distintos establecimientos educativos del país se propusieron cultivar árboles nativos, y también ocupó un rol clave en la promulgación de la reciente Ley de Educación Ambiental Integral.

En 1994, se lograron avances con el Artículo 41 de la Constitución Nacional, donde se expresa:

Todos los habitantes gozan del derecho a un ambiente sano, equilibrado, apto para el desarrollo humano y para que las actividades productivas satisfagan las necesidades presentes sin comprometer las de las generaciones futuras; $y$ tienen el deber de preservarlo. El daño ambiental generará prioritariamente la obligación de recomponer, según lo establezca la ley. Las autoridades proveerán a la protección de este derecho, a la utilización racional de los recursos naturales, a la preservación del patrimonio natural y cultural y de la diversidad biológica, y a la información y educación ambientales.

Luzzi (2000) analizó la inclusión de la EA en varios documentos nacionales vigentes al año 2000 y sostiene que la misma adquirió mayor fuerza con el mencionado artículo de la Constitución. Además, la Ley Federal de Educación N²4.195/92 y la Resolución N 37/94 del Consejo Federal de Educación introdujeron a la EA como contenido transversal (Corbetta, 2015).

En 2002, se dictó la Ley N²5.675, Ley General del Ambiente, que enuncia como uno de los objetivos de la política ambiental nacional promover cambios en los valores y conductas sociales que posibiliten el desarrollo sustentable, a través de una educación ambiental (Artículo 2); dado que la EA constituye el instrumento básico para generar en los ciudadanos y las ciudadanas valores, comportamientos y actitudes comprometidas con el desarrollo sustentable y el derecho a un ambiente sano (Artículo 14), este será un proceso continuo y permanente, sometido a constante actualización (Artículo 15). Además, la Ley General del Ambiente ratifica el COFEMA y lo sitúa como eje del ordenamiento ambiental del país y como coordinador de la educación ambiental mediante la implementación de planes o programas. Sotero (2009) realizó un análisis de las políticas públicas 
sobre EA en los países del Mercosur revisando las leyes nacionales, las específicas sobre educación ambiental, sitios de internet de instituciones de Educación y de Ambiente, y los programas nacionales de EA. El autor resalta los artículos 14 y 15 de la mencionada Ley General del Ambiente y las leyes educativas a nivel nacional, pero dice que la participación de la sociedad es tímida.

En 2006, la Ley de Educación Nacional N²6.206 sustituye a la Ley Federal de Educación. En la nueva norma, en el Artículo 89, se enuncia que el Ministerio de Educación, Ciencia y Tecnología brindará las medidas para que se favorezca la educación ambiental en todos los niveles. Otras normas federales que también contemplan la educación ambiental son la Ley de Educación Superior No 24.521 (su Artículo 3 enuncia que una de las finalidades de la Educación Superior es desarrollar las actitudes y valores que requiere la formación de personas responsables, con conciencia ética y solidaria, reflexivas, críticas, capaces de mejorar la calidad de vida y de consolidar el respeto al medio ambiente) y la Ley de Protección Integral de los Derechos de las Niñas, Niños y Adolescentes No 26.061 (su Artículo 15 dispone que los niños, niñas y adolescentes tienen derecho a una educación pública y gratuita y que esta educación debe fortalecer los valores de solidaridad, respeto por los derechos humanos, tolerancia, identidad cultural y conservación del ambiente).

Además, también existen tratados internacionales, que incluso tienen jerarquía superior a las leyes, que imponen al Estado la obligación de garantizar la educación ambiental. Por ejemplo, la Convención sobre los Derechos del Niño señala, en su Artículo 29.1, que la educación a la que todo niño, niña y adolescente tiene derecho debe estar encaminada a inculcar el respeto de los derechos humanos y del medio ambiente natural. El Acuerdo de París indica, en el Artículo 12, que los Estados deberán cooperar para mejorar la educación, la formación, la sensibilización y participación del público y el acceso público a la información, en particular sobre el cambio climático (esto luego se enmarcó en la iniciativa denominada "Acción para el Empoderamiento Climático").

Por otro lado, algunos hechos muestran que comenzó a cuestionarse el modelo de sociedad y los valores que la sustentan, como la realización del I y II Congreso de EA en la Escuela Marina Vilte de CTERA en 2004 y 2006; y el Seminario Iberoamericano de EA en 2005, organizado por el Gobierno de la Ciudad Autónoma de Buenos Aires, la Unión de Ciudades Capitales de Iberoamérica y la Unidad Temática de Ambiente de Mercociudades, donde se firmó la Declaración de Buenos Aires en Formación Ambiental. Se evidencia, además, el fortalecimiento de la EA con la creación de la Unidad de Coordinación de Educación Ambiental en el ámbito de la Secretaría de Ambiente y Desarrollo Sustentable de la Nación, y la organización del Congreso Iberoamericano de Educación Ambiental con sede en nuestro país, apuntando a promover que la EA se transforme en políticas de Estado que posibiliten transitar hacia sociedades sustentables.

Sin embargo, los conflictos socioambientales son cada vez más en la Argentina. A pesar de tener tanta legislación ambiental, cada vez es mayor la contaminación debido a la escasez de monitoreo del estado del ambiente, a la falta de control del cumplimiento de las leyes, a la inexistencia de penalidades para los que provocan los problemas. No hay relación lineal entre la formulación de la normativa y su aplicación, sino que es compleja, contextual. Por otro lado, muchos ciudadanos y muchas ciudadanas llevan adelante acciones interesantes desde una práctica 
colectiva de lucha social, aunque no tengan formación profesional específica (Telias y Canciani, 2014).

Esto evidencia la necesidad del fortalecimiento de la EA y su alcance a toda la población. Así, en octubre de 2020, el Poder Ejecutivo presentó, en el Congreso Nacional de la República Argentina, un proyecto de Ley de Educación Ambiental Integral (EAI) que propone fomentar la EA en los ámbitos formal y no formal, incorporándola en todos los niveles de la educación formal, promoviendo que las instituciones fomenten prácticas sustentables y gestionen ambientalmente sus recursos. El proyecto fue creado entre los Ministerios de Ambiente y Desarrollo Sostenible y de Educación, en consenso con las provincias mediante el Consejo Federal de Medio Ambiente (COFEMA).

El 28 de marzo de 2021 la Cámara de Diputados sancionó y giró al Senado dicho proyecto, que lo aprobó de manera unánime, el 14 de mayo de 2021, al momento de finalizarse este artículo. Dicha ley propone una EAI como un proceso permanente y transversal con el objeto de generar conciencia ambiental y una formación ciudadana para el ejercicio del derecho a un ambiente sano (Cámara de Senadores de la Nación, 2021). La ley establece que el Ministerio de Ambiente y Desarrollo Sostenible de la Nación, las jurisdicciones provinciales, la Ciudad Autónoma de Buenos Aires y el COFEMA tendrán la facultad de implementar la Estrategia Nacional de Educación Ambiental Integral (ENEAI) y las Estrategias Jurisdiccionales de Educación Ambiental Integral (EJEAI) para planificar estratégicamente la educación ambiental integral en todos los ámbitos de la educación formal, no formal, Tecnologías de Información y Comunicación (TIC) y medios de comunicación. Los principios de esta ley son: el abordaje interpretativo y holístico para el manejo de problemáticas ambientales desde el pensamiento crítico y resolutivo; el respeto de la biodiversidad; la equidad, el respeto, la inclusión y la justicia, entre los seres humanos y con la naturaleza; la igualdad de género; el reconocimiento de la diversidad cultural; la participación y formación ciudadana; el cuidado del patrimonio natural y cultural; el abordaje de las problemáticas ambientales de manera integral; la educación en valores; el pensamiento crítico que promueva el cuestionamiento de los modelos vigentes; y la concientización sobre el derecho a un ambiente sano. Además, establece la importancia de la profesionalización de los recursos humanos en la temática, la elaboración de materiales y repositorios de experiencias. Su aplicación será responsabilidad compartida entre el Ministerio de Ambiente y Desarrollo Sostenible y el Ministerio de Educación, en articulación con el COFEMA y el Consejo Federal de Educación (CFE), y esperemos que se articule, complemente y genere sinergias positivas con la Ley Yolanda.

\section{Análisis de la Ley Yolanda}

La Ley Yolanda (Ley No 27.592) debe su nombre a Yolanda Ortiz, quien fue la primera secretaria de Recursos Naturales y Ambiente Humano de Argentina, en 1973. Además, fue la primera mujer en ocupar ese cargo en América Latina. La norma fue debatida en el Senado en la sesión del 8 de octubre de 2020, luego se aprobó en la Cámara de Diputados el 17 de noviembre y fue publicada 
en el Boletín Oficial el 15 de diciembre. Su objetivo principal, que se desprende del Artículo 1, es garantizar la formación integral en ambiente, con perspectiva de desarrollo sostenible y con especial énfasis en cambio climático, para las personas que se desempeñen en la función pública. Es decir, establece que todos los empleados y todas las empleadas del Estado, en todos sus poderes, niveles y jerarquías, deben recibir una capacitación obligatoria en materia ambiental (Artículo 2). Básicamente, podría compararse con la norma que se conoce como Ley Micaela (Ley No 27.499), de 2019, que impone una obligación similar de capacitación para funcionarios y funcionarias públicas, pero en materia de género.

A lo largo de la ley Yolanda, se indica que la formación debe incorporar las dimensiones de sensibilización y de transmisión de conocimientos. Además, se incluye el enfoque participativo para que instituciones científicas especializadas en la materia y las organizaciones de la sociedad civil puedan intervenir en el diseño de los lineamientos generales. Las temáticas mínimas de la capacitación abarcan el cambio climático, la protección de la biodiversidad, la eficiencia energética, la economía circular, el desarrollo sostenible y la normativa ambiental vigente.

Según la norma, las capacitaciones deberían comenzar a impartirse dentro del año de la entrada en vigencia de la ley. Será cada organismo estatal el encargado de garantizar la implementación de esta formación para sus trabajadoras y trabajadores, tomando como base los lineamientos generales que hará la Autoridad de Aplicación (con la participación de la sociedad civil y entidades científicas). Además, la Autoridad de Aplicación deberá certificar la calidad de las capacitaciones que elabore e implemente cada organismo, y tendrá la posibilidad realizar modificaciones y sugerencias para su mayor efectividad. En términos de acceso a la información, se establece que la Autoridad de Aplicación debe difundir el grado de cumplimiento de la ley en los distintos organismos. A través del Decreto 270/2021, publicado en el Boletín Oficial el 28 de abril, se designó al Ministerio de Ambiente y Desarrollo Sostenible como Autoridad de Aplicación y se facultó específicamente a la Subsecretaría Interjurisdiccional e Interinstitucional del Ministerio a dictar las normas aclaratorias y/o complementarias que resulten necesarias para el efectivo cumplimiento de la Ley Nº 27.592 (esta Subsecretaría tiene entre sus objetivos planificar y coordinar la inserción de la política ambiental en los Ministerios y en las demás áreas de la Administración Pública Nacional, como así también intervenir en la implementación de la política de educación ambiental).

Cabe destacar que esta norma se aplicará en los organismos nacionales, de competencia federal; no obstante, se invita a la Ciudad Autónoma de Buenos Aires y a las provincias a adherir a esta ley para que esta clase de capacitación también llegue a empleados y empleadas estatales que dependen de otra jurisdicción distinta a la nacional. Al momento de finalizar este capítulo, algunas provincias ya se habían adherido, entre ellas: Chaco, Tucumán, Neuquén, Mendoza y Córdoba.

Con respecto a los debates en el Congreso, la senadora Eugenia Catalfamo destacó la necesidad de "poder promover una verdadera conciencia ambiental entre toda la sociedad civil, pero por sobre todas las cosas en aquellos agentes públicos que todos los días tienen que pensar y definir políticas públicas en torno a este tema" (2020, p. 55). La senadora Gladys González, por su 
parte, afirmó que, cuando se trabaja la agenda ambiental, se lucha por "la equidad, por la igualdad, por la justicia, por el acceso a derechos humanos y por el desarrollo" (2020, p. 57) y comparó y complementó esta agenda con la temática de género. Asimismo, la senadora Clara Vega recordó la conexión de esta ley con los Objetivos de Desarrollo Sostenible de la Agenda 2030 (2020). Otro punto presente en los discursos fue la transversalidad de la materia ambiental, que hace necesaria esta ley.

En la Cámara de Diputados, por ejemplo, la diputada Gabriela Cerruti retomó este punto al señalar que no se puede enfocar en el trabajo, la salud, la educación y el buen vivir si no se piensa primero en el ambiente, y consideró que cuando se discute acerca del ambiente se trata, en definitiva, de cómo convertir a este mundo en un lugar más justo (2020). La diputada Nilda Carrizo afirmó que el cuidado del ambiente debe ser la principal responsabilidad para quienes toman decisiones públicas, advirtió la falta de perspectiva ambiental en el Poder Judicial argentino e insistió con que la justicia ambiental es necesaria para que haya justicia social (2020). El diputado Felipe Álvarez, por su parte, consideró que existe una obligación de tomar conciencia de las consecuencias ambientales que generan las decisiones y acciones de quienes desempeñan funciones públicas y cuestionó la falta de conciencia ambiental a la hora de planificar políticas públicas durante décadas (2020). A su vez, recordó la problemática de la basura y la disposición final de residuos y alertó sobre la falta de coordinación entre los sectores público y privado. Sobre el final, advirtió que, si bien la norma es un buen comienzo, la formación ambiental no solo debe implicar a las autoridades, sino que se precisa la toma de conciencia en estos temas por parte de todos los sectores de la sociedad (2020). El diputado Nicolás Del Caño, en su turno, criticó la hipocresía y sostuvo que, si bien desde algunos partidos políticos se celebra esta ley de capacitaciones, al mismo tiempo, se impulsa un modelo extractivista, de fracking y de megaminería (2020). En esta misma línea, la diputada Romina Del Plá también cuestionó que más que un problema de educación, se trata de problemas de intereses.

Por último, vale destacar que, en sus intervenciones, tanto diputados y diputadas como senadores y senadoras valoraron el trabajo articulado y consensuado entre los distintos bloques y fuerzas políticas y el impulso generado desde las organizaciones de la sociedad civil y, en particular, de los grupos de jóvenes. La votación en la Cámara de Senadores fue unánime mientras que, en Diputados, se registraron 213 votos afirmativos, dos abstenciones y un voto negativo.

En cuanto a las críticas de Del Caño y Del Plá, quienes se abstuvieron en la votación, consideramos lo siguiente: coincidimos en que la educación ambiental no va a resolver todos los problemas, pero estamos convencidos de que es un paso en la dirección correcta. La formación para empleados y empleadas estatales en estos temas ayudará a modificar los intereses, valores y actitudes de las personas; precisamente de eso se trata la educación ambiental y la educación en derechos humanos. Por supuesto que la militancia, las asambleas ambientalistas, las luchas y las movilizaciones también pueden ser herramientas útiles para contribuir a un cambio de paradigma, pero sostenemos que estas medidas deben ser complementarias y no excluyentes. 


\section{Conclusiones}

La alfabetización ambiental apunta a conseguir una ciudadanía responsable, solidaria, crítica, con la capacidad de tomar decisiones informadas y de participar en la solución de las problemáticas para mejorar la calidad de vida de las personas. Esta educación requiere de un enfoque transversal, interdisciplinario y que trabaje sobre problemáticas locales y cotidianas.

Todos estos requisitos y objetivos son compartidos también por la educación en derechos humanos, que busca promover el respeto universal y efectivo de todos los derechos humanos (incluido el derecho a un medio ambiente sano) y contribuir a la prevención de los abusos y el empoderamiento de las personas para poder transformar y mejorar el mundo en el que vivimos. Ambas iniciativas, que se ven influenciadas por el pensamiento freireano, pretenden entonces estimular el pensamiento propio y crítico, e impulsar la modificación de ciertas conductas y valores para alcanzar sociedades más justas. No se debe perder oportunidad de recordar la estrecha interrelación entre estas propuestas y destacar las posibles sinergias que se pueden conseguir si se trabajan a la par; ojalá la aplicación de la Ley Yolanda incorpore esta perspectiva de derechos humanos.

Las discusiones sobre educación ambiental a nivel internacional comenzaron hace ya 50 años y nuestro país ha adoptado numerosas normas que consagran el derecho de las personas de recibir esta clase de formación y la obligación del Estado de brindarla. Sin embargo, aún reconociendo que existen numerosos avances, tanto a nivel nacional como a nivel mundial, todavía no se toman los parámetros ambientales, de desarrollo sostenible ni de cambio climático como ejes de todas las decisiones que se adoptan tanto desde el Estado como desde el sector privado. La Ley Yolanda podrá ser una herramienta que ayude a cubrir esta falencia, pero seguramente no podrá alcanzar este objetivo por sí sola. Su sinergia con la reciente Ley de EAl podría ser muy importante para lograr objetivos concretos en la construcción de una sociedad ambientalmente alfabetizada. Esperamos que la implementación de la norma contribuya a aumentar la sensibilización ambiental de las personas tomadoras de decisiones y que esto redunde en una mayor protección de la naturaleza y en los objetivos sociales señalados anteriormente. No obstante, aunque son muy valiosas estas capacitaciones como parte de la educación informal, esta clase de conocimientos deben incorporarse integralmente en la currícula de todas las escuelas y universidades, para así alcanzar a toda la población, y no solo a empleados y empleadas estatales. La ley de EAl es un paso importante en esa dirección. De hecho, este enfoque de formación sobre esta temática para toda la ciudadanía también significaría una formación temprana para los futuros agentes de la administración pública nacional y tomadores de decisiones, que se complementará luego con la Ley Yolanda cuando ingresen a cumplir sus funciones. A su vez, estas acciones educativas deberán ser complementadas con actividades culturales y de militancia. En cualquier caso, fundamentalmente, todo paso hacia la dirección correcta debe ser valorado. 


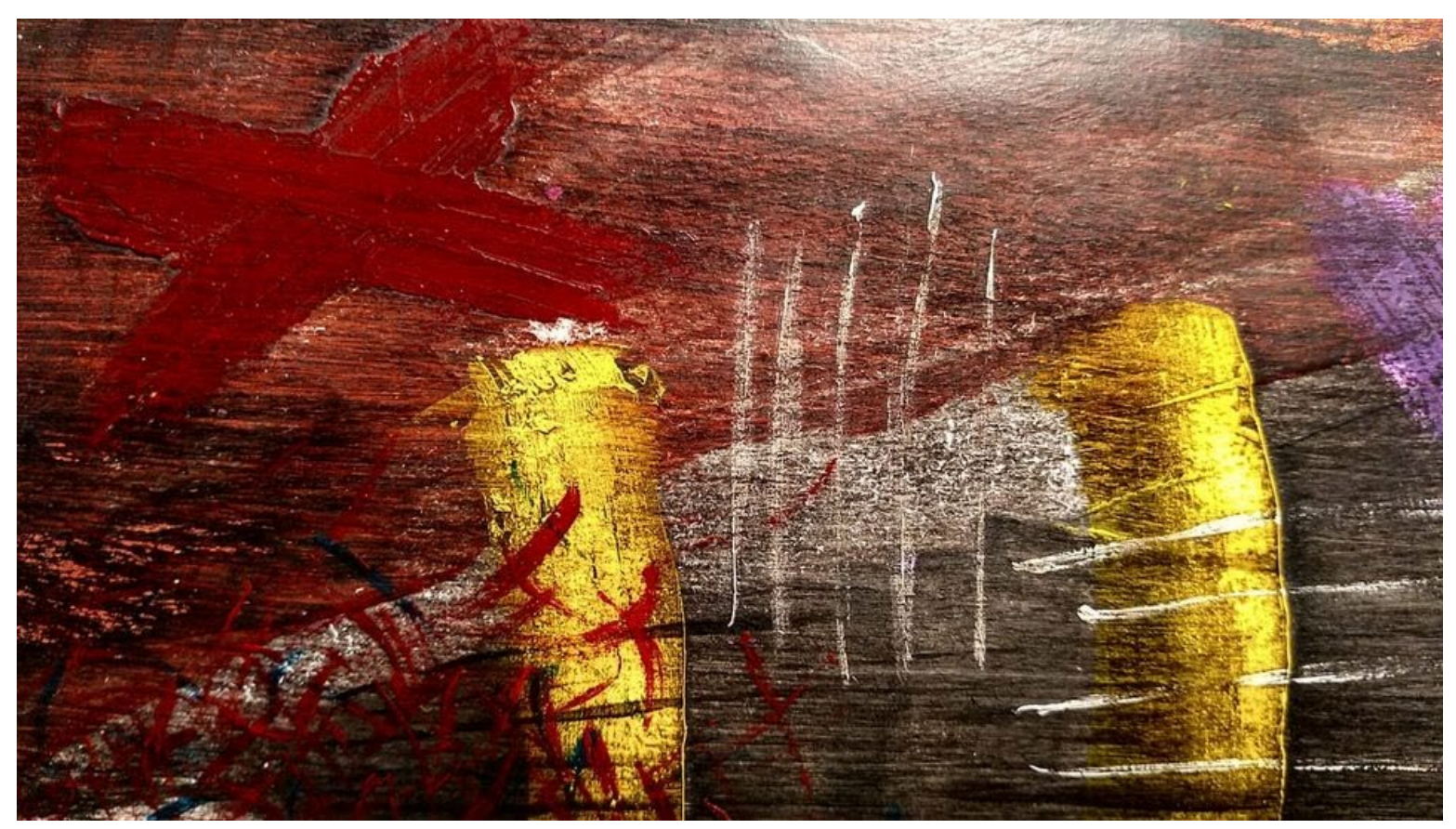

Arenas movedizas, acrílico. Matías Sapegno

\section{Bibliografía}

Asamblea General de las Naciones Unidas. (2020). Informe del Relator Especial sobre la cuestión de las obligaciones de derechos humanos relacionadas con el disfrute de un medio ambiente sin riesgos, limpio, saludable y sostenible, A/75/161. ONU.

Asamblea General de las Naciones Unidas. (2015). Transformar nuestro mundo: la Agenda 2030 para el Desarrollo Sostenible, A/RES/70/1. ONU.

Báez Tobar, O. (2015). Pensamiento, saber y racionalidad ambiental. ANALES de la Universidad Central del Ecuador, 373, 127-143. http://revistadigital.uce.edu.ec/index.php/anales/article/view/1342/1311.

Carmona Lara, M. C. (2010). Derechos Humanos y Medio ambiente. En J. Carmona Tinoco y J. Hori Fojaco (Coords.), Derechos Humanos y Medio Ambiente (pp. 1-34). Universidad Nacional Autónoma de México. Comisión Económica para América Latina y el Caribe [CEPAL]. (2018). Acuerdo Regional sobre el Acceso a la Información, la Participación Pública y el Acceso a la Justicia en Asuntos Ambientales en América Latina y el Caribe. Naciones Unidas. https://www.cepal.org/es/acuerdodeescazu consultado el 26 de septiembre de 2020.

Comisión Interamericana de Derechos Humanos (CIDH). (2019). Informe Empresas y Derechos Humanos: Estándares Interamericanos, OEA/Ser.L/V/IICIDH/REDESCA/INF.1/19.

Corbetta, S. (2015). Pensamiento ambiental latinoamericano y Educación Ambiental. Voces en el Fénix, 43, 160-

167. https://www.vocesenelfenix.com/sites/default/files/numero_pdf/fenix43\%20baja.pdf.

Corte Interamericana de Derechos Humanos (Corte IDH). (2017). Medio ambiente y derechos humanos. Opinión Consultiva 23/17.

Corte Interamericana de Derechos Humanos (Corte IDH). (2020). Caso Comunidades Indigenas Miembros de la Asociación Lhaka Honhat (Nuestra Tierra) Vs. Argentina. Sentencia de Fondo, Reparaciones y Costas.

Fisas, V. (1998). Cultura de paz y gestión de conflictos. Icaria/Unesco. 
García, D. y Priotto. G. (2009). Educación ambiental: Aportes políticos y pedagógicos en la construcción del campo de la Educación Ambiental. Jefatura de Gabinete de Ministros, Secretaría de Ambiente y Desarrollo Sustentable de Argentina.

Conzález Gaudiano, E. (2012). La ambientalización del currículum escolar: breve recuento de una azarosa historia. Revista del currículum y formación del profesorado, 16(2), 16-24. https://recyt.fecyt.es/index.php/profesorado/article/view/43677.

Honorable Senado de la Nación Argentina. (2021). Período $139^{\circ}$ - $5^{\circ}$ Reunión - $4^{\circ}$ Sesión Especial - 13 de mayo de 2021. https://www.senado.gob.ar/parlamentario/sesiones/busquedaTac.

Honorable Cámara de Diputados de la Nación Argentina. (2020). Período $138^{\circ}$ - $14^{\circ}$ Reunión - $12^{\circ}$ Sesión Ordinaria (Especial Remota) - 17 de noviembre de 2020. https://www.hcdn.gob.ar/secparl/dtaqui/.

Honorable Senado de la Nación Argentina. (2020). Diario de Sesiones: Período 1380, $17 a$ Reunión, $16^{a}$ Sesión Especial, 8 de octubre de 2020. https://www.senado.gob.ar/parlamentario/sesiones/busquedaTac.

Instituto Interamericano de Derechos Humanos. (IIDH). (2013). El Derecho a la Educación en Derechos Humanos en las Américas.

Luzzi, D. (2000). La EA formal en la educación general básica argentina. Revista Tópicos en Educación Ambiental, 2(6), 35-52.

Magendzo, A. (2001). La pedagogía de los derechos humanos. IPEDEHP. https://docplayer.es/13643923-Lapedagogia-de-los-derechos-humanos.html.

Mas, M. V. y Vignolo, V. (2017). Experiencias educativas: Enseñanza de la Biología y TIC. Editorial Académica Española.

Medici, A. (2018). Articulación de docencia-investigación-extensión para una práctica pedagógica crítica en la enseñanza de los Derechos Humanos. Revista Derechos en Acción, 77), 126-145.

Morin, E. (2011). La vía. Para el futuro de la humanidad. Paidós.

Pérez Aguirre, L. (2007). Si digo educar para los derechos humanos. Revista DEHUIDELA, 715), 49-56.

Pinto, M. (2010). La enseñanza de Derechos Humanos en la Universidad de Buenos Aires. Academia: Revista sobre enseñanza del Derecho, 8(16), 9-21.

Ronconi, L. (2017). La enseñanza en derechos humanos en las Facultades de Derecho en Argentina: desafíos pendientes. Revista Pedagogía Universitaria y Didáctica del Derecho, 4(1), 5-37.

Roth, C. (1992). Environmental literacy: Its roots, evolution and directions in the 1990s. ERIC Clearinghouse for Science, Mathematics and Environmental Education. http://files.eric.ed.gov/fulltext/ED348235.pdf.

Sauvé, L. (2003). Perspectivas curriculares para la formación de formadores en Educación ambiental. / Foro Nacional sobre incorporación de la Perspectiva Ambiental en la Formación técnica y profesional. San Luis Potosí, México.

Sauvé, L. (2010). Educación científica y Educación Ambiental: un cruce fecundo. Enseñanza de las Ciencias, 28(1), 5-18.

Sauvé, L. (2013). Saberes por construir y competencias por desarrollar en la dinámica de los debates socioecológico. Integra Educativa, 15(3), 65-87.

Sotero, J. P. (2009). Políticas Públicas en Educación Ambiental: rol y responsabilidad del Estado en su promoción. VI Congreso Iberoamericano de Educación Ambiental. San Clemente del Tuyú, Argentina.

Telias, A., Canciani, M. L., Sessano, P. y Alvino, S. (2014). Construcción ciudadana y alfabetización ambiental: las prácticas ciudadanas sustentables como prácticas educativas. En A. Telias, M. L. Canciani, P. Sessano, S. Alvino y A. Padawer, La educación ambiental en la Argentina: actores, conflictos y políticas públicas (pp. 75-88). La Bicicleta Ediciones. 
Telias, A. y Canciani, M. L. (2014). Educación ambiental, currículum y formación docente. En A. Telias, M. L. Canciani, P. Sessano, S. Alvino y A. Padawer, La educación ambiental en la Argentina: actores, conflictos y políticas públicas (pp. 157-174). La Bicicleta Ediciones.

Tibbitts, F. (2017). Evolution of Human Rights Education Models. En M. Bajaj (Ed.), Human Rights Education: Theory, Research, Praxis (pp. 69-95). University of Pennsylvania Press.

Vilches, A. y Gil Pérez, D. (2012). La educación para la sostenibilidad en la Universidad: el reto de la formación del profesorado. Revista de currículum y formación del profesorado, 16(2), 25-43. 